\title{
Die Stadt als Assemblage: Neue Perspektiven für die Stadtplanung durch die Actor-Network- Theorie?
}

\section{The City as Assemblage: Does the actor-network theory offer new perspectives for urban planning?}

\section{FRANK ECKARDT, WEIMAR}

Zusammenfassung: Städte zeichnen sich durch das Zusammenspiel von gebauter und sozialer Welt aus. In der Stadtforschung wird durch die Rezeption der Actor-Network-Theorie (ANT) seit einigen Jahren versucht, diese Erkenntnis zur Erweiterung der Handlungsperspektiven für die Stadtplanung zu nutzen. Dabei soll mit dem Rückgriff auf den Begriff der Assemblage der offensichtliche Gegensatz zwischen Materialität und Gesellschaft überwunden werden. In diesem Beitrag soll anhand von vier internationalen Studien aus der angewandten Stadtplanungsliteratur diskutiert werden, inwieweit sich durch die Urban-Assemblage-Forschung neue Perspektiven für die Stadtplanung gewinnen lassen. Anhand von vier thematischen Handlungsfeldern (Citizenship, Rekonfiguration des Gebauten, Materialität und Gemeinschaft, Soziale Bewegungen und Stadtplanung) soll aufgezeigt werden, in welcher Weise ANT rezipiert wird und ob sich tatsächlich neue Perspektiven für die Stadtplanung ergeben können. Im Ergebnis wird aufgezeigt, dass es in der Tat durch eine veränderte Sicht auf Stadtplanung, die Beziehungen zwischen der sozialen und nichtmenschlichen Stadt integriert, in diesen Studien gelingt, neue Optionen für eine demokratisierende Stadtpolitik anzudeuten. Allerdings wird zugleich deutlich, dass diese empirischen Studien auch auf Defizite des Urban-Assemblage-Ansatzes hinweisen und insbesondere die Berücksichtigung der politischen und materiellen Dimension des Städtischen noch weiter verbessert werden muss.

Schlagwörter: Assemblage, Actor-Network-Theorie (ANT), Citizenship, Stadtforschung, Stadtplanung 
Abstract: Cities are characterized by the interplay between the built and the social world. In urban research, the Actor Network Theory (ANT) has received attention since many years, so that this knowledge can be used to improve the ability of action in urban planning. By referring to the concept of assemblage, the relationships between materiality and society can be restored. This contribution aims to discuss the results of four international studies from the applied urban planning literature, in which new perspectives for urban planning can be gained through the theory of urban assemblage research. On the basis of four thematic fields of action (citizenship, reconfiguration of the building, materiality and community, social relationships and urban planning), the aim is to explain how ANT is adapted and whether new perspectives for urban planning can be considered. The result shows that these studies do indeed succeed in the sense of providing a new perspective on urban planning, which integrates the relationships between the social and non-human city, with new options for a democratizing urban policy. Apparently, however, these empirical studies identify deficits of the urban assemblage approach and indicate that future research must address especially more intensively the political and material dimensions of the city.

Keywords: assemblage, actor network theory, citizenship, urban planning, urban research

\section{Einleitung}

Städte zu erklären ist ein schwieriges Unterfangen. Schon Max Weber tat sich schwer damit zu definieren, was eigentlich eine Stadt ausmacht (Kemper 2012). Immer wieder wird dabei von einzelnen Aspekten städtischen Lebens und aktuellen Tendenzen in der Stadtentwicklung auf allgemein gültige Aussagen zum Leben in der Stadt geschlossen (vgl. Breckner, Göschel und Matthiesen 2020; Eckardt 2009). In der Stadtforschung ringen unterschiedliche Auffassungen um Geltung, die sich in der Regel auf übergeordnete theoretische Erklärungen aus der Soziologie, Architektur, Philosophie und den Kulturwissenschaften beziehen. Die Stadt wird als ein Beispiel gesehen, auf welches deduktiv die allgemeineren Annahmen über die Gesellschaft, Geschichte oder die Anthropologie des Menschen angewandt werden (eine Übersicht in Eckardt 2014). Derartigen zumeist disziplinär legitimierten Theorien über die Stadt steht das Unbehagen jener Forscher/innen gegenüber, die der Stadt als solche einen eigenständigen theoretischen Wert einräumen wollen und die die Stadtforschung als ein genuin transdisziplinäres Forschungsfeld verstehen, in dessen Mittelpunkt die Frage stehen soll, wie Städte angesichts der vielen ökologischen und gesellschaftlichen Herausforderungen zu planen sind. 
In einer solchen transdisziplinären Stadtforschung (Ward und Jayne 2017) wird die Stadt nicht nur als Gegenstand empirischer Forschung betrachtet, anhand dessen sich theoretische Annahmen überprüfen lassen. Vielmehr werden Städte als eigenständige Orte der konzeptionellen Produktion von Wissen verstanden (Parker 2000). Die Materialität der Stadt gerät deshalb neuerdings (wieder) in den Aufmerksamkeitsfokus der Stadtforschung und dies soll auch konzeptuell aufgearbeitet werden. Das kann etwa bedeuten, von der Annahme der Existenz eines lokalen Wissens auszugehen, das aufgrund der jeweils spezifischen Materialität der einzelnen Städte genuin ortsabhängig ist: Wanne-Eickel ist nicht New York. Allgemeine Aussagen über Städte müssten für beide Orte relevant sein, in Wirklichkeit ist aber offensichtlich, dass vermeintlich objektives Wissen durch die Heterogenität des Städtischen in Frage gestellt wird. Spezifische lokale Kontexte produzieren mehr urbanes Wissen, als dies über eine Kategorisierung von Orten als „Stadt“ erreicht werden kann. Dementsprechend ist die lokale, auf die je spezifische Materialität fokussierende „Bottom-up“-Perspektive in der Stadtforschung, insbesondere in der angewandten Form und mit Bezug auf die Stadtplanung, oftmals wichtiger als abstrakt-kategorisierende Ansätze. Die angewandte Stadtforschung hat es daher auch mit einer anderen Form von Theorie zu tun, die sich nach Robert A. Beauregard (2008; 2013) eher als „Theoretisierung“ verstehen lässt, die ein Handeln und Planen in einer konkreten Stadt zum Ziel hat. Interessant ist daher die Frage, ob sich durch die Anwendung neuer Ansätze, um die es in diesem Artikel gehen soll, ein anderes Theorie-Verständnis etablieren lässt, welches jenseits akademischer Diskurse auch eine alltagsweltliche Bedeutung haben kann. Das Feld der Stadtplanung, in der eine enge Verquickung von Stadtforschung und Anwendungsbezug immer schon gegeben ist, soll hierfür im Folgenden als Beispiel dienen.

Theoretische Ansätze, die sich um eine Einbeziehung der lokalen und anwendungsorientierten Wissensproduktion bemühen, sind für die Stadtforschung besonders interessant (Storper und Scott 2016). Der Begriff der Assemblage, wie er in der Actor-Network-Theorie (ANT) eingeführt wurde, hat in der neueren Beschäftigung mit der Stadt Anklang gefunden. Vor allem durch einen Sammelband von Ignacio Farías und Thomas Bender (2011) wurde die Verschmelzung zweier bis dahin getrennter Beschreibungs- und Analysediskurse durch den Begriff der „Urban Assemblage“ angestrebt. Damit erhofft man sich, Phänomene in der Stadt besser beobachten und erklären zu können, als dies mit dem bisherigen Vokabular der Stadtforschung gelingt. 
Der Begriff der „Urban Assemblage“ geht zurück auf die Actor-Network-Theorie (einführend: Belliger und Krieger 2006), die sich ab den 1980er Jahren im Bereich der Science and Technology Studies (STS) entwickelt hat und die von der grundlegenden Erkenntnis ausgeht, dass die Welt netzwerkartig verfasst ist und dass Vernetzung auch für die Beziehungen zwischen der Welt im umfassenden Sinne und menschlichen Gesellschaften entscheidend ist. Somit wird das Soziale als Bestandteil von Netzwerken gesehen, durch die es mit der Welt in vielfältiger Weise verknüpft ist. Zentral ist dabei der Gedanke, dass auch Dinge (Aktanten) in Netzwerke mit Menschen eingebunden sind (vgl. Law 2006). Der Grundgedanke der Vernetzung von dinglichen Objekten und Menschen hat in der Stadtforschung in unterschiedlicher Weise Anklang gefunden, wobei der verschieden definierte und verwendete Begriff der „Urban Assemblage“ eine prominente Rolle eingenommen hat. Die Unterschiede in Konzeptionen der Urban Assemblage betreffen die Frage, ob der Begriff lediglich methodologisch zu verstehen ist, als eine spezifische Form der Beschreibung der Mensch-Welt-Beziehungen, oder aber ob mit dem Begriff eine ontologische Grundannahme bezeichnet wird, der zufolge Vernetzungen von Menschen mit der dinglichen Seite der Welt unaufhebbar und für Gesellschaften konstitutiv sind. Als eine Art Konsensdefinition, in der diese Uneindeutigkeit aber erhalten bleibt, kann man die Urban Assemblage als Begriff verstehen, der die Beziehungen zwischen Beziehungen in der Stadt (als relationalem Raum) wie im physisch-geographischen Territorium bezeichnet. Die Fluidität und Fixierung von Beziehungen zwischen Raum, globalen und lokalen Kontexten, Strukturen und Handlungen gerät somit in den Blick. Urban Assemblages sind „equally sensitive to the role of relational and territorial geographies, of fixity and flow, of global contexts and place-specificities, of structural imperatives and embodied practices“ (McCann und Ward 2011, 175).

Dieser innovative Ansatz hat schnell zu der Diskussion geführt, ob das gegebene Versprechen, gegenwärtige Phänomene der Stadtentwicklung besser erklären zu können, wirklich eingelöst wird. Insbesondere die Reaktion von Neil Brenner (Brenner, Madden und Wachsmuth 2011), einem der profiliertesten Vertreter einer kritischen Stadtsoziologie, hat aufgezeigt, dass die ANT-inspirierte Assemblage-Perspektive die Art und Weise, wie Städte verstanden und untersucht werden sollten, grundsätzlich hinterfragt. Brenner lehnt die ontologische Ausdeutung des Assemblage-Begriffs ab, da diese kein geeignetes Instrumentarium zum Verständnis derjenigen gesellschaftlichen Rahmenbedingungen biete, in denen städtische Räume und lokal eingebet- 
tete soziale Kräfte positioniert sind. Nach Brenner, Madden und Wachsmuth (2011) blendeten Assemblage-Ansätze die widersprüchlichen und hierarchischen sozialen Beziehungen und institutionellen Formen des Kapitalismus aus und verhinderten dadurch Kritik an tatsächlich bestehenden sozialen Beziehungen und institutionellen Arrangements. Eine ähnliche Diskussion hat der die ANT weitgehend verteidigende Beitrag von Alexa Färber (2014) in der deutschen Stadtforschung ausgelöst.

Inzwischen liegen weitere Fallstudien vor (Blok und Farías 2016; Kärrholm 2013; Nakajima 2013; Dovey und Fisher 2014; Grossmann und Haase 2016; Lancione 2016a, 2016b; Wang, Oakes und Yang 2016; Figueiredo 2016), die es erlauben, die Frage nach der Erklärungskraft der ANT in der angewandten Stadtforschung zu diskutieren. Nach einer Rekapitulation der bisherigen Diskussionen um die Bedeutung der Assemblage-Forschung für die kritische Stadtforschung soll in diesem Beitrag eine Auseinandersetzung mit neueren Studien erfolgen. Dabei wird davon ausgegangen, dass sich die Frage nach der Anwendbarkeit der ANT am Grundverständnis kritischer Stadtforschung (vgl. Eckardt 2014, 89ff.) entscheidet, welche eine Steigerung des emanzipatorischen Potentials theoretischer wie praktischer Diskurse über Stadtplanung anstrebt. Die Fragestellung, die hier verfolgt werden soll, lautet daher: Kann mit einem ANT-Ansatz ein Erkenntnisgewinn für eine an der Stadtplanung orientierte Stadtforschung erzielt werden und werden dadurch gleichzeitig zusätzliche emanzipatorische Handlungsoptionen erkennbar? Dementsprechend sollen Fallstudien untersucht werden, die sowohl versuchen, mit Hilfe der ANT die Handlungsperspektiven der Stadtplanung zu erweitern, als auch dem normativen Anspruch der Stadtplanung, demokratisierend zu wirken, entsprechen wollen. Ausgewählt wurden daher Fallstudien, die eine deliberative oder partizipatorische Ausrichtung der Stadtplanung analysieren, in der soziale Bewegungen und die Zivilgesellschaft als relevante Planungsakteure gesehen werden. Obwohl inzwischen umfangreiche Studien aus der ANT-orientierten Forschung (Guggenheim 2008, 2016; Marres 2012; Wilde 2020) vorliegen, ist die Anzahl von Studien, die den genannten Anforderungen entsprechen, noch relativ klein. Es kann aber auf vier Studien - aus den Niederlanden (Koster 2015), den USA (Sheehan und Vadjunec 2012), Australien (Oakley 2014) und Spanien (Marrero-Guillamón 2013) - zurückgegriffen werden, die originären Planungskontexten entstammen und daher Anwendungsprobleme berücksichtigen müssen. 


\section{Der Begriff der Urban Assemblage}

Die Notwendigkeit, den für die angewandte Stadtforschung neuen Begriff der „Urban Assemblage“ einzuführen, hat sich aus einer länger anhaltenden Unzufriedenheit vieler Forscher/innen ergeben, die die sozialwissenschaftliche Erforschung der Zusammenhänge zwischen Stadt und Gesellschaft insbesondere hinsichtlich ihrer materiellen Substanz als untertheoretisiert betrachtet haben (vgl. Eckardt 2016). Aus dem Grunde haben verschiedene Autor/innen vor allem seit den 2000er Jahren Ansätze formuliert, die sich nicht auf eine Beschreibung der Stadt als entweder gebaut oder gelebt, entweder materiell oder sozial oder auf ähnlich duale Konzeptionen beschränken. Die triadische Konzeption des Raums bei Lefebvre (Schmid 2010) hat diese Simplifizierungen ebenso zu überwinden versucht wie andere Autor/innen, die von übergeordneten theoretischen Konzeptionen ausgehen. Zu letzteren gehören Soziologen wie Christopher Mele (2000) und Simon Parker (2000), die sich schon vor den Urban-Assemblage-Studien darum bemühten, eine diskurs-, handlungs- und erfahrungssoziologische Perspektive auf die Stadt mit der Betrachtung ihrer materiellen Konstruktion in Einklang zu bringen. Beide Autoren betonen, dass das Verständnis von Handlungen nicht wiederum zu neuen dualen Kategorisierungen im Sinne von Handeln versus Reden/Denken/Planen führen darf. Sie gehen vielmehr von einer Gleichzeitigkeit von Raumproduktion und Raumwahrnehmung aus, die konzeptionell zusammengedacht werden müssen. Mele und Parker sind nicht als Vorläufer der Assemblage-Theorie zu verstehen, haben aber durch ihre Arbeiten bewirkt, dass das Thema der materiellen Seite der Stadt in der Stadtsoziologie überhaupt wieder aufgegriffen wurde (Müller 2017). Mele und Parker nutzen dabei teilweise ältere, aber weniger einflussreiche Traditionen der Stadtsoziologie, die sich gegenüber den in der amerikanisch-europäischen Diskussion dominierenden struktursoziologischen Ansätzen nicht haben durchsetzen können. Sie lassen sich eher in den theoriegeschichtlichen Kontext der Material-Culture-Forschung einordnen, wobei insbesondere Meles Arbeiten zur Gentrifizierung von New York von der Vereinbarkeit von kritischer Stadt- und kritischer Diskursforschung ausgehen.

Städtische Assemblages beschreiben weniger bestimmte Objekte, die in einer festgelegten Beziehung zueinander beschrieben und untersucht werden können. Interessanter ist die Prozesshaftigkeit, mit der städtische Akteure in assemblierte Situationen eintreten, diese deassemblieren und dann wiederum reassemblieren, indem bestehende Beziehungen zwischen 
Objekten wie zwischen Objekten und Akteuren zerlegt und wiederum neu zusammengefügt werden. Dies ist nur deshalb möglich, weil es eigentlich nie nur eine Assemblage gibt, sondern Städte durch die permanente Co-Produktion verschiedener Beziehungen gekennzeichnet sind, die durch kreatives Handeln immer wieder neu verknüpft werden können. Entscheidend ist dabei, dass diese Neuverknüpfungen nicht durch Inbesitznahme geschehen müssen, sondern auch durch Interaktionen der Komponenten auf den intermediären Ebenen (Agencies) möglich sind. Städte sind in dieser Hinsicht als ein Produkt der Geschichte dieser Interaktionen zu betrachten und beherbergen durch diese historische Pfadabhängigkeit auch je unterschiedliche Potentiale für neue Prozesse der De- und Reassemblierung. Wie McFarlane (2011, 654) betont, hat die Erforschung städtischer Assemblages deshalb auch mehr mit dem Thema Wandel zu tun, als dies für die klassische ANT gilt, die ihren Fokus auf die Stabilität von Netzwerken richtet. Die Stabilität der immer neu veränderten Assemblages wird vielmehr durch einen Prozess der stetigen Wieder-Ausrichtung auf vorhandene Strukturen von Macht, Wissen, Diskursen, Rationalitäten und Dispositiven hergestellt, wobei diese Wieder-Ausrichtung in einem Kontext der Ungleichverteilung von Macht, Ressourcen und Wissen erfolgt.

Die Forschungen zur Urban Assemblage haben sich von Beginn an nicht primär mit konzeptionell-theoretischen Fragen auseinandergesetzt; auch die theoretische Nähe zu den die ANT prägenden Arbeiten von Bruno Latour kann nicht als übertrieben groß bezeichnet werden. Colin McFarlane gibt in seinem Aufsatz über das Wohnen als Assemblage (2011) der Freiheit in der Interpretation von ANT-Theoretikern wie Latour den entsprechenden Raum, indem er vorschlägt, sich nicht weiter mit unterschiedlichen Begriffsdefinitionen aufzuhalten, sondern von Assembling statt Assemblage zu sprechen, weil es ihm um eine theoretische Sensibilisierung zugunsten einer verbesserten empirischen Fokussierung und nicht um eine abstrakte Begriffsdiskussion geht. Das drückt sich auch in einem entsprechenden Stadtverständnis aus, wobei gilt: „The city [...] is not just inhabited but [...] is produced through inhabiting“ (651). Damit wird ein Stadtverständnis aufgegriffen, welches wiederum handlungstheoretisch fundiert und beispielsweise auch in Nigel Thrifts und Ash Amins Buch „Cities. Reimagining the Urban“ (2011) präsent ist, an das wiederum Farías und Bender (2011) anknüpfen. Die Anschlussfähigkeit an die ANT besteht für McFarlane im Hinblick auf die „non human actors“, die in der bisherigen Stadtforschung unberücksichtigt geblieben seien. Die Aufnahme der Assemblage-Perspektive ist für ihn 
deshalb auch eher eine Korrektur von bisherigen, verengten Perspektiven der Stadtforschung, durch die etwa die Mobilität und Translokalität des Wohnens in Sicht kommen, die ansonsten in der „bounded, ,earthy“ baggage of connotations that dwelling carries" $(2011,652)$ nicht erscheinen.

Es ist also möglich, von einer Vereinbarkeit von Assemblage Thinking und Critical Urban Studies auszugehen, wobei die historischen Prozesse sozialer Ungleichheiten nach wie vor als grundlegend angesehen werden. Mit der Rede von der Stadt als Assemblage kann sowohl eine Orientierung als auch ein Objekt bezeichnet werden: Während eine Assemblage-Orientierung eine ontologische Sichtweise begründen will, in der die sozialen, politischen, kulturellen oder ökonomischen Zusammenhänge als ein Beziehungsgeflecht verstanden werden, das eine gemeinsame Erforschung von Praxis und Materialität verlangt, kann die Assemblage auch als eine Objekt-Forschung begriffen werden, in der die Beziehungen zwischen Objekten im Mittelpunkt stehen. Diese große Varianz innerhalb der Assemblage-Stadt-Forschung verhindert eine orthodoxe Festlegung auf ein zu anspruchsvolles Forschungsdesign. Sie erschwert zugleich eine Aussage darüber, worauf es denn in den Assemblage-Studien letztlich ankommt. Arbeitet man die theoretischen Annahmen heraus, mit denen die unterschiedlichen Ansätze operieren, wird man zu dem Ergebnis kommen, dass die Objekt-Assemblage wohl wesentlich weiter verbreitet ist als die ontologische Variante der AssemblageOrientierung.

Festzuhalten bleibt, dass sich der Ansatz der Urban Assemblage nicht als eine Engführung eines vorformulierten metatheoretischen Konzepts verstehen lässt, sondern eher die soziomateriellen Aspekte von Stadt und Urbanität zu thematisieren versucht, die zwischen makrogesellschaftlichen Prozessen und materiellen Objekten und Artefakten auf intermediären Ebenen der Interaktion angesiedelt sind und die es zu erforschen gilt. Dabei werden Netzwerke, wie sie für die ANT so wichtig sind, ebenso in Augenschein genommen wie Agencies, Atmosphären oder Sozialtechnologien. Diesbezüglich liegen auch theoretische Anleihen bei anderen philosophischen Konzeptionen, insbesondere bei Deleuze, nahe.

\section{Einwände der kritischen Stadtsoziologie}

Deutet man die Assemblage-Ansätze in der dargestellten Weise, scheinen die gedanklichen Brücken zu einer Stadtforschung, die sich als Kritik an bestehenden sozialen Ungleichheiten versteht und diese offenlegen will, of- 
fensichtlich zu sein. Die Urban-Assemblage-Studien sind allerdings entweder von wichtigen Stadtforschern wie David Harvey oder Manuel Castells überhaupt nicht rezipiert oder doch insgesamt negativ bewertet worden. Im schon erwähnten Text von Brenner, Madden und Wachsmuth (2011) wird dabei eine doppelte Gegenargumentation aufgebaut. Zum einen wird eine fundamentale Ablehnung formuliert, die in erster Linie als eine politische verstanden werden muss: „Instead, we must continue to seek out the ingredients - intellectual and political - for a critical imagination that is oriented towards the possibility of a radical different type of worldwide space." (Brenner, Madden und Wachsmuth 2011, 238) Es gelte, die globale urbane Welt im Sinne von Marx bei den Wurzeln zu packen. Der Ausgangspunkt ist dabei die Konstatierung eines profunden globalen Wandels, der sich nicht als ein rein städtisches Phänomen beschreiben lässt. Stattdessen stehen geoökonomische Restrukturierungen im Vordergrund, die „large scale, long term trends“ (ebd.) zum Ausdruck bringen. Obwohl die Autoren die Notwendigkeit der Reflexion vorhandener Analyse-Konzepte und Theorien nicht verleugnen, halten sie es nicht für notwendig, in einem poststrukturalistischen Sinne die „grand narratives“, etwa vom Wachstum und von der Entwicklung von Städten (vgl. Brockow-Loga und Eckardt 2020), in Frage zu stellen. Wie angesichts einer solchen Vorabfestlegung die sich selbst zugeschriebene Offenheit für neue Denkansätze Wirklichkeit werden kann, wird von den Autoren nicht thematisiert.

Gegen den Ansatz der Urban Assemblage wird zweitens emphatisch argumentiert, indem dieser zunächst vor allem als eine Art Methode („guiding sensibility or research orientation“, Brenner, Madden und Wachsmuth 2011, 238) akzeptiert, aber die darüber hinausgehenden Ambitionen der Theorie-Innovation abgelehnt werden. Vorgeworfen wird den Assemblage-Forscher/innen ihre ungenaue Terminologie, die keine präzisen empirischen und normativen Parameter bereitstelle. Brenner, Madden und Wachsmuth bestehen auf der Festlegung eines Forschungsobjektes $(2011,230)$ und fragen, ob hier ein neuer Metadiskurs initiiert wird, der Ansätze der politischen Ökonomie ablösen soll. Durch die Unübersichtlichkeit der Assemblage-Studien irritiert, ordnen sie diese nach einem relativ einfachen Muster, wobei sie die vorhandenen Studien und Publikationen entweder als empirische, methodologische oder ontologische Forschungen verstehen. Anschließend vergleichen sie deren Erträge mit denjenigen, die sie aus Studien ziehen können, die sich dem eigenen metatheoretischen Diskurs der politischen Ökonomie verpflichtet sehen. Bei diesem vergleichenden, ergebnisorientierten Vor- 
gehen geht allerdings das Besondere der Assemblage-Forschung verloren, die ihren soziomateriellen Fokus als Erweiterung makrogesellschaftlicher Strukturanalysen und nicht als deren bloße Anwendung verstanden wissen will. Mit Bezug auf McFarlanes Studie zu informellen Siedlungen in Indien behaupten die Autoren, dass die dichte Beschreibung der Soziomaterialität keine neuen Einsichten liefere, weil soziomaterielle Studien über Marginalisierung in Megastädten etwa schon für ähnliche Siedlungen in Südamerika vorliegen. Der soziomaterielle Fokus, so der Vorwurf, lässt die spezifischen geohistorischen Kontextbedingungen weitgehend außer Betracht. Daraus abgeleitet bleibt für die Kritiker/innen der Urban-Assemblage-Forschung die Schwachstelle bestehen, dass keine konkreten Akteure oder Aktanten benannt oder in ihrer politischen Konstitution hinreichend wahrgenommen werden, die eine tatsächliche Veränderung bewirken könnten.

Wenn man die kontroverse Diskussion um den Stellenwert der Urban Assemblage kurzfassen will, dann wird vor allem die Berücksichtigung übergeordneter geopolitischer Transformationsprozesse wie die Etablierung einer neoliberalen und postfordistischen globalen „urban order“ von den Kritiker/innen eingefordert. Auf der operationellen Ebene wird nachgeordnet gefragt, ob eine hinreichend transparente und rigide Methodologie entwickelt worden ist. Mit Bezug auf diese beiden Kritiken (globale Kontextualisierung und Politikverständnis sowie Methodologie) sollen nun vier planungsbezogene Studien dargelegt werden, die explizit an die Urban-Assemblage-Forschung anschließen.

\section{Urban Assemblage und Stadtplanung}

\section{Citizenship als Assemblage}

In seiner Studie über den sozialen Wohnungsbau in den Niederlanden führt Martijn Koster (2015) eine historische Analyse durch, bei der er sich auf die Veränderungen des letzten Jahrzehnts konzentriert. Hierbei führt er den Begriff der Assemblage mit zwei Intentionen ein. Zum einen will er damit zeigen, dass nicht nur Regierungspolitik zum Entstehen neuer Wahrnehmungsschemata von „guten“ und „schlechten“ Bürger/innen geführt hat, sondern dass diese in einem Prozess der Assemblage durch staatliche wie nichtstaatliche Akteur/innen zustande gekommen sind. Der Assemblage-Ansatz ist gewählt worden, „to avoid downplaying the role of non-state actors in governance assemblages" (Koster 2015, 225). 
Zum anderen will der Artikel demonstrieren, dass auch unvorhergesehene Ereignisse wie der Bankrott von Wohnungsunternehmen oder die Skandalisierung von Korruption betrachtet werden müssen, wenn man die Wieder-Ausrichtung (,alignment“) der Wohnungspolitik als einen Prozess des Wandels der allgemeinen Governance in den Niederlanden versteht. Hervorgehoben wird die bestehende Pfadabhängigkeit, die Koster durch die schon vorhandenen politischen Ideologien und Regulatorien gegeben sieht. Der soziomaterielle Impetus der Assemblage-Forschung wird von ihm sozioräumlich übersetzt und dient so seiner anthropologischen Feldforschung im benachteiligten Utrechter Stadtteil Overvecht.

Koster bettet seine Studie in Überlegungen zu globalen Veränderungen durch die Dominanz neoliberaler Politik ein, die zu einer Bevorzugung der sozial aufsteigenden Bürger/innen und von Eigentum führe. Durch den Assemblage-Ansatz will er aber auch aufzeigen, dass die Definition von Bürgerschaft (Citizenship) nicht mehr nur rein national-territorial erfolgt, sondern auch durch andere territoriale Entitäten wie den sogenannten sozialen Brennpunkten beeinflusst wird. Die lokale Polis ist in diesem Sinne nicht mit dem Nationalstaat identisch und folgt eher globalen Vorstellungen von einem aktiven, selbstverantwortlichen und partizipierenden Bürger. Durch den ANT-Ansatz kann Koster zeigen, dass sich das lokale Verständnis von Citizenship nicht lediglich aus der Ableitung nationaler Vorgaben und Gesetze ergibt, sondern stattdessen räumliche Praktiken vor Ort Citizenship durch konkrete Handlungen interpretieren.

Methodisch macht der Autor in diesem Beitrag nur ansatzweise von seiner vorhandenen anthropologischen Kenntnis des Stadtteils Gebrauch. Nichthumane Aktanten im eigentlichen Sinne kommen bei ihm kaum in den Blick, da es bei seinen Beobachtungen in erster Linie um das Verhalten von Menschen zueinander geht und der Stadtteil quasi nur die Bühne darstellt, auf der das Verhalten der Bewohner/innen beobachtet werden kann. Konsequenterweise fordert Koster $(2015,225)$ weitere Studien „for a more rigorous academic analysis of how these assemblages gain and change shape, and the different stakes that emerge within these assemblages".

\section{Materialität und Gemeinschaft}

Als geradezu gegensätzliches Beispiel einer ANT-inspirierten Studie kann man die Arbeit von Rebecca Sheehan und Jacqueline M. Vadjunec (2012) verstehen, in der sich Politik nur im Sinne eines „butt the government out“ wiederfindet und die im Kontext amerikanischer Regionalisierung verbleibt. 
Dabei wäre eine weitergehende Kontext-Verortung naheliegend, handelt es sich doch bei dem untersuchten Cimarron County in Oklahoma um eine Gegend, die von den globalen Prozessen der geopolitischen Neuordnung durch die Ausrichtung der Ökonomie auf den Weltmarkt existentiell betroffen ist. Obwohl Cimarron County als sterbende Stadt massiv durch den Einwohnerverlust bedroht ist, finden die Autorinnen in ihren auf Oral History, semistrukturierten Interviews und teilnehmender Beobachtung basierenden Untersuchungen eine Art von Gemeinschaftsgeist vor, der sich nicht hinreichend durch das Faktum der verlorengehenden Stadt verstehen lässt.

Stattdessen ermöglicht ihnen eine weitergehende Interpretation des Assemblage-Ansatzes, zunächst eine Entkopplung zwischen der räumlichen Konstitution der Kleinstadt und der Gemeinschaft („community“) zu beobachten: Die Gemeinschaft ist mehr als nur eine räumlich konstruierte soziale Ebene, die sich durch Anwesenheit und Nähe quasi natürlich ergeben hat. Vielmehr verstehen die Autorinnen sie als eine Art von emotionaler Assemblage, die sich als „being-in common“, als ein Netzwerk von Menschen, Glaube und Landschaft beschreiben lässt. Entscheidend ist dabei, dass dieses Zusammengehörigkeitsgefühl die Materialisierungen der kulturellen Landschaft und ihre aktive Bearbeitung existentiell miteinschließt. Als Coping-Mechanismus für „common success and common misery“ (Sheehan und Vadjunec 2012, 932) dient diese Konstruktion der Gemeinschaft aus nichthumanen/landschaftlichen Aktanten und Menschen auf Grundlage eines assemblierten Gefühls auch dazu, sich gegen einen als fremd erfahrenen „common sense“ abzugrenzen. Die Autorinnen dokumentieren, dass Bedrohungen der materiellen und natürlichen Lebensgrundlagen die Bewohner/ innen dazu zwingen, sich auf die Gemeinschaft zu verlassen, um mit dem Leben im Niemandsland fertig zu werden. In der Rekonstruktion der Narrative, mit denen sie sich als in die Kulturlandschaft eingebettet beschreiben, dominiert die Figur des kollektiven Körpers, in dem die Landschaft und die Bewohner-Gruppe als miteinander verbunden erscheinen. Auf der Basis dieses Befundes zur Assemblage eines Gemeinschaftsgefühls darf angenommen werden, dass eine solche Form der landschaftsinklusiven Selbstwahrnehmung nicht lokal begrenzt ist und viele Menschen auch in anderen Regionen der USA ähnlich empfinden: „By identifying circulations of a variety of sometimes very different actants and actor-networks, we were also able to consider ,community" not as a ,thing' with assumed qualities and geographical boundaries, but rather as a broader set of ideas and actions.“ (ebd.) Die Autorinnen schließen mit dem Hinweis, dass es für Politiker sehr wichtig ist 
zu verstehen, wie diese komplexen Actor-Network-Beziehungen sich gestalten, um mit diesen Gemeinschaften verhandeln und innerhalb ihrer handeln zu können.

Diese Studie, die sich mit einem induktiven Forschungsdesign der Assemblage-Theorie nähert, betrachtet die ANT-Theorie vor allem als eine erweiterte Möglichkeit zur Beschreibung von Befunden, die sich im Rahmen der Community-Study-Tradition der bisherigen Feldforschung nicht angemessen thematisieren lassen. Anders als bei Koster wird die materielle Dimension insofern hervorgehoben, als die Landschaft und auch verschiedene Lokalitäten in ihrer symbolisch-konkreten Gestalt, etwa in der Bezeichnung von Bergen und Seen, in der Schilderung der eigenen Biographie auftreten und als Orte der Assemblierung des Gemeinschaftsgefühls erkenntlich werden. Die Selbst-Platzierung in einer Landschaft erzeugt in diesem Sinne erst die Gemeinschaft.

\section{Rekonfiguration des Gebauten}

Während die amerikanische Studie formale Planungsprozesse weitgehend außer Acht lässt, fokussieren die beiden folgenden Beispiele auf ebendiese. Im engeren Sinne planungstheoretisch orientiert ist dabei aber nur Susan Oakleys Artikel (2014). Der planungsorientierte Ansatz wird durch einen auf Objekte fokussierten Zugang begründet, der sich auf Port Adelaide als Beispiel für eine Waterfront-Revitalisierung kapriziert. Oakley bewertet die dazu vorhandene Forschungsliteratur als ambivalent und stellt „kritischen“ Analysen, die in solchen Projekten den Ausdruck von neoliberalen Restrukturierungen der globalen Ökonomie sehen, jene gegenüber, die diese Projekte auch als Erfolgsgeschichten betrachten, bei denen es Regierungen in bereits benachteiligten Gegenden gelingt „to house, employ and ensure quality environments“" (Oakley 2014, 172).

Eine kritische Urban-Assemblage-Forschung verlangt der Autorin zufolge, dass Möglichkeiten des Wandels aufgezeigt werden, wobei zunächst dasjenige urbane Problem identifiziert werden muss, auf das die Planung eine Antwort geben soll, damit über letztere dann in einem politischen Prozess entschieden werden kann. In der Erforschung dieses Prozesses können laut Oakley die Begriffe der Deskription, der Agency sowie der Imagination der Assemblage produktiv genutzt werden. Die Deskription soll hier (im Sinne von McFarlane 2011, 210) auch die historische Entwicklung städtischer Ungleichheiten in den Blick nehmen, die durch Veränderungen der Beziehungen zwischen Politik, Wirtschaft, Gemeinschaften und Aktivismus 
im kapitalistischen Kontext geprägt ist. Mit dem Begriff der Agency soll die Materialität nicht nur als Objekt, sondern auch als etwas verstanden werden, das durch Prozesse der Stadtplanung zum Erreichen bestimmter politischer Ziele gestaltet werden kann. Zum Beispiel wurden Dokumente oder Werbematerialien erstellt, um zu kommunizieren und bestimmte Aktionen der Planung anzuleiten und Akteur/innen zu gemeinsamen Handlungen zu organisieren. Mit der Imagination der Assemblage wird schließlich darauf hingewiesen, dass die Vorstellung eines „Rechts auf Stadt“ mit einer allgemeinen Politik der Anerkennung zu verbinden ist. Neues städtisches Wissen soll dadurch generiert werden, dass Kollektive organisiert werden, die eine Vielzahl von Themen und Anliegen und zugleich eine Reihe von Identitätsformationen - Klasse, Rasse, ethnische Zugehörigkeit, Geschlecht und Sexualität - verbinden.

Oakley (2014) beginnt ihre Argumentation mit einer Kontextualisierung des deindustrialisierten und verwahrlosten Hafengeländes, auf dem ein bauliches Vorhaben realisiert werden soll, welches zugleich das Entstehen Tausender Jobs und die Revitalisierung des Gebiets zum Ziel hat. So weit stellt die Darstellung des Planungsprozesses eine relativ konventionelle Beschreibung dar, die aber von der Autorin durch einen besonderen Fokus auf das Branding der „Newport Quays“ sowie auf die überhöhte Skalierung der geplanten Gebäude erweitert wird. Die Aufnahme dieser Aspekte des Planungsprozesses werden von Oakley bereits als Teil der „Critical Urban Assemblage“-Forschung gesehen. Anschließend wird die globale Finanzkrise ab 2008 als „Reassembling“ eingeführt, die zu einem teuren Ende der Kooperation und einer Rückgabe der Planung an den Staat mit der Ankündigung neuer, ,nachhaltigerer Kooperationen geführt hat. Dies soll unter anderem durch eine verstärkte Beteiligung der Öffentlichkeit gelingen, die bis dato außen vor geblieben war. In der Zusammenfassung wird die Verwendung der Perspektive einer kritischen Urban Assemblage als Rahmen „for a more comprehensive and inclusive policy and practice option“ (Oakley 2014, 185) angesehen. In welcher Weise dies geschehen könnte, wird nur durch Bezugnahme auf „urban planning that builds on the ,local' in the broadest sense“ (ebd.) vage angedeutet. Im Gegensatz zur Betonung der meisten Assemblage-Interpretationen und im Kontext eines selbstdeklarierten Zugangs zum Baulichen überraschend, bleibt gerade die materielle Dimension nur auf die Frage des Branding bezogen, das hauptsächlich am Beispiel von Hinweisschildern illustriert wird. 


\section{Soziale Bewegungen und Stadtplanung}

Isaac Marrero-Guillamón (2013) hat mit seinen ethnographischen Arbeiten über die Konflikte, die sich durch die Besetzung der Can-Ricart-Fabrik in Barcelona ergeben haben, ebenfalls einen theoretischen Anschluss an die Urban-Assemblage-Forschung hergestellt. Marrero-Guillamón greift stärker als die drei anderen Studien auf eine eigene Lesart verschiedener Traditionen der ANT zurück, wobei er auch als Einziger die starke These Latours zur Rolle der nichtmenschlichen Aktanten und des Parlaments der Dinge (Latour 2001; Greif 2005) reflektiert. Nach seinem Verständnis stellt Latour vor allem die Privilegierung formaler (Entscheidungs-)Strukturen in Frage und hebt die Bedeutung verschiedener Räume der Politik hervor, die auf den „different forms of being together" (Marrero-Guillamón 2013, 418) beruhen und ihrerseits in der Produktion von Kollektiven, Objekten und Bifurkationen untersucht werden müssen. Marrero-Guillamón ist sich dabei bewusst, dass er mit einer solchen Interpretation der ANT teilweise an der ursprünglichen Intention der Assemblage-Forschung vorbeigeht und die Materialität des Netzwerkes sich so eher verflüchtigt. Ungeachtet dessen soll aber durch den Fokus auf kreative Innovationen („new collectives, new political objects, and new conditions of possibility“ (ebd.)) ein Einklang mit den Ursprüngen der ANT hergestellt werden, die sich zuerst mit der Frage nach der Generierung und Innovation von Wissen beschäftigte.

Diskursive Operationen und direkte Aktionen, die Produktion und Kommunikation eines neuen politischen Objekts (die Fabrik) und die damit einhergehende Produktion neuer rhetorischer und akademischer Narrative stellen sich als ein vernetztes Vorhaben, als eine Assemblierung des Zusammenseins dar, welches sich im Kampf um Anerkennung neu ausrichtet und eine soziale Ausdifferenzierung produziert, die sich als Reassembling weiter fortsetzt. Durch eine strikte Anwendung der ANT, so der Autor, wäre insbesondere diese Phase nicht erklärbar, weil sie keine Multiplizierung der „common world“ vorsieht. Erkennbar wird aber, dass die Arbeit der Aktivist*innen nicht lediglich als Rekomposition einer bereits existierenden Welt zu deuten ist, sondern vielmehr durch ihre kreative Konstruktion ein neuer politischer Prozess entsteht. 


\section{Fazit}

Die Anwendung eines kritischen Urban-Assemblage-Ansatzes in der praxisnahen Stadtforschung bietet der emanzipatorischen Stadtplanung einen Rahmen, der für eine inklusive Stadtpolitik und Planungspraxis neue Optionen bereithält. Die Perspektive der ANT erschließt eine Reihe von lokal bedingten Handlungspraktiken, die sich an unvorhergesehene politische, soziale und ökonomische Veränderungen anpassen können. Angesichts der Tatsache, dass Stadtplanung langfristige, oft auf Jahrzehnte hin angelegte Projekte verfolgt, ist ein solches Verständnis der menschlichen Interaktion mit der gebauten physischen und nichtmenschlichen Welt gegenüber schematischen Planungsphilosophien im Vorteil, die sich nur auf die soziale Welt beziehen. Planung lässt sich somit relational denken (Kurath und Bürgin 2019), indem Artefakte, Wissensformen und Praktiken gedanklich miteinander verbunden werden. Planungstheoretisch würde sich anbieten, die ANT als ein Beispiel für solche Theorien der Stadtplanung zu diskutieren, die bislang konzeptionell sehr lose unter der Kategorie der „soft planning“ (Allmendinger 2009; Haughton 2010) versammelt werden, wobei Stadtplanung dabei nur undeutlich als prozesshaft und nicht objekt- und produktorientiert verstanden wird. Die Begrifflichkeit der ANT hingegen könnte der konzeptionellen Undeutlichkeit entgegenwirken, wobei die vier hier behandelten Schwerpunkte (Citizenship/Gemeinschaft, Materialität, Rekonfiguration des Gebauten, soziale Bewegungen) zumindest ansatzweise als analytische Fokusse zu betrachten sind.

In der Praxis ergeben sich, wie die angeführten Studien zeigen, durch die Erweiterung der angewandten Stadtplanungsforschung um Aspekte der nichtmenschlichen Umwelt Perspektiven, welche die Planung einer für verschiedene Gemeinschaften nachhaltigen, integrativen und lebenswerten städtischen Umgebung unterstützen können. Gefordert ist eine kreative Stadtplanung, die sich mit dem „Lokalen“ im weitesten Sinne und damit sensibel mit der Vergangenheit, Gegenwart und Zukunft des jeweils spezifischen Ortes auseinandersetzt. Es lässt sich in diesen Studien auch die Herausbildung eines Konsenses darüber beobachten, wie die ANT-orientierte Urban-Assemblage-Forschung zentrale Begriffe wie die „Assemblage“ selbst definiert. Dabei werden konzeptionelle Unschärfen teilweise bewusst in Kauf genommen und für eigene Interpretationsansätze genutzt. Die Anwendung der ANT in diesen stadtplanerischen Studien bedeutet zugleich keineswegs, dass die Ansätze der kritischen Stadtforschung außer Acht gelassen werden. Vielmehr ist eine Verschmelzung von Theorie-Horizonten zu konstatieren, 
wobei die Bedeutung globaler Transformationen der kapitalistischen Wirtschaftsordnung, wie sie die Ansätze der politischen Ökonomie hervorheben, auch im Rahmen von Assemblage-Forschungen anerkannt wird. Das Anliegen der Assemblage-Forscher/innen, in erster Linie neue Erkenntnisse initiieren zu wollen, indem sowohl konzeptionell wie methodisch auf Grundlage der ANT etwa Bezüge zu nichtmenschlichen Aktanten hergestellt werden, steht bei den hier besprochenen Studien im Vordergrund. Dass die Verknüpfung von verschiedenen theoretischen Ansätzen tatsächlich auch in anderen Fällen erkenntnisfördernd ist, kann jedoch nicht von vornherein angenommen werden und müsste im Einzelfall begründet werden (vgl. Sohn 2016).

Ausgesucht wurden die Studien, weil sie sich in impliziter und teilweise expliziter Weise einer normativen Ausrichtung von Stadtplanung verpflichtet sehen, die bewusst eine emanzipatorisch-demokratisierende Sichtweise auf Planungspraktiken und -prozesse entwickelt. Auffallend ist dabei, dass die politische Dimension der Stadtplanung teilweise gänzlich unreflektiert bleibt (Sheehan und Vadjunec 2012) oder aber nur ansatzweise adressiert wird (Koster 2015). Politik erscheint hier als Prozess ohne personalisierte Entscheidungshandlungen. In diesem Sinne folgen die Studien einem Politikverständnis, das sich eher als Gesellschaftspolitik im weiteren oder deliberativen Sinne von Stadtplanung (vgl. Fischer 2003; Healey 2010) beschreiben ließe. Andererseits gelingt es der australischen wie der spanischen Fallstudie, gesellschaftliche und räumliche Prozesse des Wandels in Beziehung zu einem emanzipatorisch-demokratisierenden Politikwechsel zu setzen und zugleich im Einklang mit Annahmen über die veränderten Vorgaben der globalen Ökonomie zu analysieren (vgl. auch McCann, Roy und Ward 2013). Dennoch wird offensichtlich, dass eine Politisierung der Assemblage im Ansatz konzeptionell nicht zwingend angelegt ist und sich planungstheoretisch aus den Beschreibungen der Reassemblierung keine politischen Schlussfolgerungen ableiten lassen. Auffallend ist auch, dass in der Assemblage-Forschung zwar Kritik an Prozessen der Stadtentwicklung geübt wird, man sich zugleich jedoch nicht konstruktiv etwa auf planungssoziologische Perspektiven einlässt (vgl. Ureta 2014 oder Shand 2014).

Ernüchternd ist der Befund auch hinsichtlich der Einbeziehung der materiellen Seite stadtgesellschaftlichen Wandels, der oftmals in der Diskussion um den Stellenwert der ANT eine große Rolle spielt. Obzwar insbesondere in der Studie über die sterbenden Städte von Oklahoma sehr viel von Raum und Materialität tatsächlich ,sichtbar wird, wird die materielle Seite der Stadt doch nur im Kontext einer symbolischen Landschaft aufgegriffen. 
Die stadtplanungsorientierte ANT-Forschung müsste in der Hinsicht weiterentwickelt werden, dass auch die Interaktionen zwischen Menschen und nichtmenschlichen Objekten anschaulicher und besser erkennbar werden. Um sowohl eine gesellschaftlich-historische Kontextualisierung der jeweiligen Stadt besser vornehmen als auch empirische Prozesse der Vernetzung zwischen Akteur/innen und Aktanten genauer beobachten und zueinander in Beziehung setzen zu können, wäre im Sinne einer kritischen Planungssoziologie ein anspruchsvoller Forschungsrahmen notwendig, der zwischen der angemessenen Berücksichtigung urbaner Komplexität und dem Wunsch nach konkreter Handlungs- und Planungsorientierung die richtige Balance finden muss.

\section{Literatur}

Allmendinger, Philip. 2009. Planning theory. 1. Aufl. Basingstoke: Palgrave Macmillan.

Beauregard, Robert A. 2008. „Theorizing the city“. Annals of the Association of American Geographers Nr.1: 239-242.

Beauregard, Robert A. 2013. „The neglected places of practice“. Planning Theory \& Practice Nr.1: 8-19.

Belliger, Andréa, und David J. Krieger, Hrsg. 2006. ANThology. Ein einführendes Handbuch zur Akteur-Netzwerk-Theorie. 1. Aufl. Bielefeld: transcript.

Blok, Anders, und Ignacio Farías, Hrsg. 2016. Urban cosmopolitics: agencements, assemblies, atmospheres. 1. Aufl. London: Routledge.

Breckner, Ingrid, Albrecht Göschel und Ulf Matthiesen. 2020. „Gesamteinleitung“. In Stadtentwicklung und Stadtsoziologie, hrsg. von Ingrid Brecker, Albrecht Göschel und Ulf Matthiesen, 1. Aufl., 5-11. Baden-Baden: Nomos.

Brenner, Neil, David J. Madden und David Wachsmuth. 2011. „Assemblage urbanism and the challenges of critical urban theory“. City Nr. 2: 225-241. https://doi.org/ 10.1080/13604813.2011.568717.

Brockow-Loga, Anton, und Frank Eckardt, Hrsg. 2020. Postwachstumsstadt: Konturen einer solidarischen Stadt. München: oekom.

Dovey, Kim, und Kenn Fisher. 2014. „Designing for adaptation: the school as socio-spatial assemblage“. The journal of architecture Nr. 1: 43-63. https://doi.org /10.1080/13602365.2014.882376.

Eckardt, Frank. 2009. Die komplexe Stadt: Orientierungen im urbanen Labyrinth. 1. Aufl. Wiesbaden: Springer.

Eckardt, Frank. 2014. Lehrbuch Stadtforschung: Gegenstand und Methoden. 1. Aufl. Wiesbaden: Springer. 
Eckardt, Frank. 2016. „Technologie und Virtualität als strukturierendes Element des Sozialraums“. In Handbuch Sozialraum, hrsg. von Fabian Kessel und Christian Reutlinger, 243-258. Wiesbaden: Springer.

Färber, Alexa. 2014. „Potenziale freisetzen: Akteur-Netzwerk-Theorie und Assemblageforschung in der interdisziplinären kritischen Stadtforschung“. sub/urban Nr.1: 95-103. https://doi.org/10.36900/suburban.v2i1.109.

Farías, Ignacio, und Thomas Bender, Hrsg. 2011. Urban assemblages: how actor-network theory changes urban studies. 1. Aufl. London: Routledge.

Figueiredo, Bernardo. 2016. „Home in mobility: an exercise in assemblage thinking“. In Assembling consumption: researching actors, networks and markets, hrsg. von Robin Canniford und Domen Bajde, 1. Aufl., 77-91. London: Routledge.

Fischer, Frank. 2003. Reframing public policy: discursive politics and deliberative practices. 1. Aufl. Oxford: Oxford Univ. Press.

Greif, Hajo. 2005. „Wer spricht im Parlament der Dinge?: Über die Idee einer nicht-menschlichen Handlungsfähigkeit“. 1. Aufl. Paderborn: Mentis.

Grossmann, Katrin, und Annegret Haase. 2016. „Neighborhood change beyond clear storylines: what can assemblage and complexity theories contribute to understandings of seemingly paradoxical neighborhood development?". Urban geography 37, Nr. 5: 727-747.

Guggenheim, Michael. 2008. „Building memory. Architecture, networks and users“. Memory Studies Nr.1: 39-53.

Guggenheim, Michael. 2016. „Im/Mutable Im/Mobiles. From the Socio-Materiality of Cities towards a Differential Cosmopolitics“. In Urban Cosmopolitics. Agencements, Assemblies, Atmosphere, hrsg. von Anders Blok und Ignacio Farias, 6381. 1. Aufl. London: Routledge.

Haughton, Graham. 2010. The new spatial planning: territorial management with soft spaces and fuzzy boundaries. 1. Aufl. London: Routledge.

Healey, Patsy. 2010. Making better places: the planning project in the twenty-first century. 1. Aufl. Basingstoke: Palgrave Macmillan.

Kärrholm, Mattias. 2013. „Building type production and everyday life: rethinking building types through actor-network theory and object-oriented philosophy“. Environment and Planning Nr. 6: 1109-1124. https://doi.org/10.1068/d15312.

Kemper, Jan. 2012. „Max Weber“. In Handbuch Stadtsoziologie, hrsg. von Frank Eckardt, 31-58. 1. Aufl. Wiesbaden: Springer.

Koster, Martijn. 2015. Citizenship agendas, urban governance and social housing in the Netherlands: an assemblage approach“. Citizenship Studies Nr. 2: 214-228. http://dx.doi.org/10.1080/13621025.2015.1005951.

Kurath, Monika, und Reto Bürgin, Hrsg. 2019. Planung ist unsichtbar. Planungssoziologie zwischen relationaler Designtheorie und Akteur-Netzwerk-Theorie (ANT). 1. Aufl. Bielefeld: transcript. https://doi.org/10.14361/9783839448533001. 
Lancione, Michele. 2016a. Rethinking life at the margins: the assemblage of contexts, subjects and politics. 1. Aufl. London: Routledge.

Lancione, Michele. 2016b. „Racialised dissatisfaction: homelessness management and the everyday assemblage of difference". Transactions of the Institute of British Geographers Nr. 4: 363-375. https://doi.org/10.1111/tran.12133.

Law, John, Hrsg. 2006. Actor Network Theory and After. 1. Aufl. Oxford: Blackwell.

Latour, Bruno. 2001. Das Parlament der Dinge: für eine politische Ökologie. 1. Aufl. Frankfurt: Suhrkamp.

Marrero-Guillamón, Isaac. 2013. „Actor-Network Theory, Gabriel Tarde and the Study of an Urban Social Movement: The Case of Can Ricart, Barcelona“. Qualitative Sociology Nr. 4: 403-421.

Marres, Noortje. 2012. Material Participation: Technology, the Environment and Everyday Publics.1. Aufl. London: Palgrave Macmillan.

McCann, Eugene, Ananya Roy und Kevin Ward. 2013. „Assembling/Worlding Cities“. Urban Geography Nr. 5: 581-589. https://doi.org/10.1080/02723638.20 13.793905 .

McCann, Eugene, und Kevin Ward, Hrsg. 2011. Mobile Urbanism: Cities and Policymaking in the Global Age, 1. Aufl. Minneapolis, London: University of Minnesota Press.

McFarlane, Colin. 2011. „The city as assemblage: dwelling and urban space“. Environment and Planning Nr. 4: 649-672.

Mele, Christopher. 2000. „The Materiality of Urban Discourse: Rational Planning in the Restructuring of the Early Twentieth-Century Ghetto“. Urban affairs quarterly Nr 5: 628-648.

Müller, Anna Lisa. 2017. „Simon Parker: Urbanism as Material Discourse“. In Schlüsselwerke der Stadtforschung, hrsg. von Frank Eckardt, 93-106. 1. Aufl. Wiesbaden: Springer.

Nakajima, Seio. 2013. „Re-imagining Civil Society in Contemporary Urban China: Actor-Network-Theory and Chinese Independent Film Consumption“. Qualitative Sociology Nr. 4: 383-402. https://doi.org/10.1007/s11133-013-9255-7.

Oakley, Susan. 2014. „Understanding the Planning and Practice of Redeveloping Disused Docklands Using Critical Urban Assemblage as a Lens: A Case Study of Port Adelaide, Australia“. Planning Practice and Research Nr. 2: 171-186. https:// doi.org/10.1080/02697459.2013.858508.

Parker, Simon. 2000. „Tales of the city: Situating urban discourse in place and time“. City Nr. 2: 233-246.

Parker, Simon. 2005. Urban theory and the urban experience: encountering the city. 1. Aufl. London: Routledge.

Sassen, Saskia. 2006. Territory, Authority, Rights: From Medieval to Global Assemblages. 1. Aufl. Princeton: Princeton University Press. 
Schmid, Christian. 2010. Stadt, Raum und Gesellschaft: Henri Lefebvre und die Theorie der Produktion des Raumes. 1. Aufl. Stuttgart: Franz Steiner.

Shand, Wayne. 2014. „Urban assemblage, street youth and the sub-Saharan African city“. City Nr. 2: 257-269. https://doi.org/10.1080/13604813.2018.1451138.

Sheehan, Rebecca, und Jacqueline M. Vadjunec. 2012. „Placing community through actor-network theory in Oklahoma's No Man's Land“. Social \& Cultural Geography Nr. 8: 915-937. https://doi.org/10.1080/14649365.2012.728616.

Sandkjaer Hanssen, Gro, und Inger-Lise Saglie. 2010. „Cognitive Closure in Urban Planning“. Planning Theory \& Practice Nr. 4: 499-521. https://doi.org/10.1080 /14649357.2010.525373.

Sohn, Christophe. 2016. „Navigating borders' multiplicity: the critical potential of assemblage“. Area 48, Nr. 2: 183-189.

Storper, Michael, und Allen J. Scott. 2016. „Current debates in urban theory: A critical assessment“. Urban Studies Nr. 6: 1114-1136. https://doi.org/ 10.1177/0042098016634002.

Thrift, Nigel, und Ash Amin. 2002. Cities. Reimagining the Urban. 1. Aufl. Cambridge: Polity Press.

Ureta, Sebastian. 2014. „The Shelter that Wasn't There: On the Politics of Co-ordinating Multiple Urban Assemblages in Santiago, Chile“. Urban Studies Nr. 2: 231-246. https://doi.org/10.1177/0042098013489747.

Wang, June, Tim Oakes und Yang Yang, Hrsg. 2016. Making cultural cities in Asia: mobility, assemblage, and the politics of aspirational urbanism. 1. Aufl. London: Routledge.

Ward, Kevin, und Mark Jayne, Hrsg. 2017. Urban theory: new critical perspectives. 1. Aufl. London: Routledge.

Wilde, Jessica. 2020. Die Fabrikation der Stadt. Eine Neuausrichtung der Stadtsoziologie nach Bruno Latour. 1. Aufl. Bielefeld: transcript. https://doi. org/10.14361/9783839455371-001. 
